\title{
Defect model for the dependence of breakdown rate on external electric fields
}

\author{
K. Nordlund and F. Djurabekova \\ Helsinki Institute of Physics and Department of Physics, University of Helsinki, P.O. Box 43, FIN-00014, Helsinki, Finland
}

(Received 1 August 2011; revised manuscript received 2 April 2012; published 11 July 2012)

\begin{abstract}
We develop an analytical model for the vacuum electric breakdown rate dependence on an external electric field, observed in test components for the compact linear collider concept. The model is based on a thermodynamic consideration of the effect of an external electric field on the formation enthalpy of defects. Although strictly speaking only valid for electric fields, the model also reproduces very well the breakdown rate of a wide range of radio-frequency breakdown experimental data. We further show that the fitting parameter in the model can be interpreted to be the relaxation volume of dislocation loops in materials. The values obtained for the volume are consistent with dislocation loops with radii of a few tens of nanometers.
\end{abstract}

DOI: 10.1103/PhysRevSTAB.15.071002

\section{INTRODUCTION}

The development of high-gradient radio-frequency (rf) linear collider accelerating structures is strongly limited by vacuum microwave breakdown [1-9]. The concept "vacuum microwave breakdown" means in this context that the high electromagnetic field causes first electron and then ion emission to the vacuum, building up a plasma that makes the vacuum conductive. This is also known as vacuum arcing [10]. This arc severely changes the electromagnetic field in the accelerating structure [1] and can damage the accelerating components $[11,12]$. Experiments consistently show that, under the influence of high electric and/or magnetic fields, the structures suffer from vacuum radiofrequency breakdowns even if the electric component of the field is smaller than those, for which the electric field evaporation of surface atoms has been observed [13].

The radio-frequency breakdown is observed in several different types of linear collider components operated at frequencies of the order of $\sim 1-10 \mathrm{GHz}$, with accelerating gradients of the order of $100 \mathrm{MV} / \mathrm{m}$ and surface electric fields of the same order of magnitude [2,14,15]. The observed breakdown rates (BDR) show a very strong dependence on the accelerating gradient, typically varying between $10^{-7}$ and $10^{-1}$ breakdowns per rf pulse (bpp). The breakdowns have been observed in components fabricated from a wide range of metals, such as $\mathrm{Al}, \mathrm{Ti}, \mathrm{Cu}$, and $\mathrm{Mo}$.

Vacuum electric breakdown is also often studied under static or slowly varying electric fields in so-called "direct current" (dc) setups [16-19]. These allow for easier testing of a wide range of materials [20], and have in general shown that the dc and rf breakdowns have several similarities, such as similar breakdown surface electric fields [17,21].

Published by the American Physical Society under the terms of the Creative Commons Attribution 3.0 License. Further distribution of this work must maintain attribution to the author(s) and the published article's title, journal citation, and DOI.
PACS numbers: 52.80.Vp, 61.72.Lk, 29.20.Ej, 52.80.Mg

It has been clearly observed that the breakdown rate is different if the different accelerating gradients were used in the components. In fact, there have been many attempts to interpret BDR as a function $R_{\mathrm{BD}}=f\left(E_{\mathrm{acc}}\right)$ (here and later $R_{\mathrm{BD}}$ stands for BDR for convenient use in analytical expressions). For instance, Grudiev et al. [8] have shown that the observed breakdown rate in rf structures can be fitted well by power law functions of the form $R_{\mathrm{BD}}=a E^{x}$, where $E$ is the accelerating gradient. Also dc breakdown probabilities show a similar power law dependence [21]. Grudiev et al. pointed out, however, that this form had not been chosen on the basis of any assumption of an underlying physical mechanism [8]. Hence, a physical interpretation of the very steep dependence on rate with field remains to be determined.

In the current article, we develop a physically motivated model for the breakdown rate observed in dc conditions.

Moreover, since the breakdown rates have been observed to be similar under dc and rf conditions [21,22], we also apply it to breakdown rate data in rf accelerating structures. Our model reproduces a wide range of literature data organized as $R_{\mathrm{BD}}\left(E_{\mathrm{acc}}\right)$ with the comparable quality of the fit as using the power law in [8], under both dc and rf conditions.

\section{MODEL FOR BREAKDOWN RATE}

\section{A. Model for dc electric field breakdown}

In dc setups used to examine breakdown phenomena $[16,18,21]$, the external potential leads to an electric field $E$ at the inside surface of the components. This field will, in turn, induce a charge on the surfaces, and hence cause a stress on the material [23,24]. From basic electrodynamics [25], one obtains that the external stress on the sample $P$ is given by

$$
P=-\varepsilon_{0} E^{2},
$$

where $\varepsilon_{0}$ is the dielectric constant of vacuum. Note that the stress is always tensile as the force $\left(\vec{F}_{L}=q_{\text {surf }} \vec{E}\right)$ acting on 
the charged surface is independent on the direction of the field and always directed from the surface [24].

We have shown previously that one possible mechanism for the onset of electrical breakdown is the growth of tips under the electric field by dislocation-induced mass transport to the surface $[18,24]$. If we assume that this dislocation-based mechanism (or a mechanism based on other dislocation reactions or crystallographic defects) is mainly responsible for the triggering of breakdown, we can consider the process from the thermodynamics point of view [26,27]. In thermodynamic equilibrium, the Gibbs free energy change $[26,28]$ when a defect is formed can be written

$$
G^{f}=H^{f}+T S^{f, \text { tot }},
$$

where $H^{f}$ is the formation enthalpy, and $S^{f \text {,tot }}$ the total formation entropy of the defect [29]. The formation enthalpy consists of the formation energy $E^{f}$ and a term proportional to the internal pressure (stress) $\sigma$ in the crystal

$$
H^{f}=E^{f}+P \Delta V=E^{f}-\sigma \Delta V
$$

(since $P=-\sigma$ ), where $\Delta V$ is the relaxation volume of the defect. The formation entropy can be divided into a vibrational and configurational part, $S^{f, \text { tot }}=S^{f \text {,vib }}+S^{f \text {,con }}$. Minimization of the configurational entropy [26] leads to an expression for the defect concentration [29-32]

$$
\begin{aligned}
c & =g e^{S^{f} / k_{B}} e^{-H^{f} / k_{B} T} \\
& =c_{0} e^{-E^{f} / k_{B} T} e^{\sigma \Delta V / k_{B} T},
\end{aligned}
$$

where $k_{B}$ is Boltzmann's constant and $g$ is the number of crystallographically equivalent permutations of a given defect structure. The notation has been simplified by rewriting $S^{f}=S^{f \text {,vib }} . c_{0}$ is a frequently used prefactor that includes the $g$ and $S^{f}$ terms $[29,32]$.

The relaxation volume of interstitial-type defects has a positive value $(\Delta V>0)$. Strictly speaking, both $\sigma$ and $\Delta V$ are direction-dependent tensors and thus the product of these quantities will have a complex shape. We will, however, focus for simplicity on a product of two scalar values not taking into account possible directional differences due to the crystal structure, since we do not aim presently at a quantitative description of the process for specific defects.

Since the pressure applied to the material in the considered case is a tensile stress with $P<0$ and $\Delta V$ as mentioned above is positive, the product $\sigma \Delta V$ will have a positive sign. The enthalpy of formation of the defect of this type $H^{f}=E^{f}-\sigma \Delta V$ clearly decreases with increasing stress, provided the defect relaxation volume does not change. A decreasing $H^{f}$ leads to an increasing defect concentration [Eq. (5)], i.e., interstitial-type defect formation becomes easier with increasing tensile stress.
If the breakdown is caused by dislocations (or other defects), it is reasonable to assume that the breakdown rate is proportional to the dislocation concentration $c$. Then inserting Eq. (1) into Eq. (5) (omitting the vector notation), grouping terms that are not dependent on the electric field, and inserting a prefactor $a^{\prime}$, we obtain

$$
\begin{aligned}
R_{\mathrm{BD}} & =a^{\prime} c_{0} e^{-E^{f} / k_{B} T} e^{\varepsilon_{0} E^{2} \Delta V / k_{B} T} \\
& =a e^{\varepsilon_{0} E^{2} \Delta V / k_{B} T} .
\end{aligned}
$$

The prefactor $a^{\prime}$ is necessary since $R_{\mathrm{BD}}$ is a macroscopic quantity and hence will depend on also purely macroscopic quantities such as the size of the system subject to the electric field. To simplify the expression, we introduce $a=a^{\prime} c_{0} e^{-E^{f} / k_{B} T}$. In the current context we use $a$ as a free parameter, adjusted by fitting the functional shape to the experimental results, in order to follow the main functional behavior with electric field $\propto f\left[\exp \left(E^{2}\right)\right]$ or temperature $T$.

\section{B. Relation to $\mathrm{rf}$ conditions}

Although the model presented above is strictly valid only for electric fields, it is of interest to attempt to apply it also for the rf accelerating structures. In these the breakdowns are related to both electric $E$ and magnetic $B$ field components of the electromagnetic wave.

Experimentally the maximum breakdown rate is observed to be related to the peak value of a quantity referred to as the modified Poynting vector [8]. This and other studies [9] indicate that both $E$ and $B$ play a role in the breakdown process. However, $E$ at the maximum Poynting vector is only $\sim 20 \%$ lower than at maximum electric field, and it has already been observed that there is a strong correlation between the electric field and breakdown rate (see e.g. [2,4,7-9,21,22,33]). Following these works, at this stage of the model development we analyze the breakdowns as a function of $E$ field only.

We note that by analysis of the local stress at the surface from both electric and magnetic fields, it should be possible to extend the model to explicitly include both components of the rf fields. Moreover, if the local stress is known everywhere in an rf accelerating structure, one could apply the model as an integral average over the surface. However, such calculations are beyond the scope of the current paper, where the aim is mainly to present the basic concept of a possible relation between stress and breakdown rate.

In terms of using electric field, it is important to note that breakdown rate data is frequently provided as a function of the accelerating gradient $E_{\text {acc }}$ rather than the surface electric field $E$ used in the model. These quantities are, however, related to each other, and typically the maximum surface electric field in a component $E=\eta E_{\text {acc }}$, where $\eta$ typically is $\approx 2[2,15,34]$. Thus the breakdown rate can be rewritten as

$$
R_{\mathrm{BD}}=a e^{\varepsilon_{0}\left(\eta E_{\mathrm{acc}}\right)^{2} \Delta V / k_{B} T}
$$




\section{Summary of model}

Equation (6) is the central result of the model. It predicts that if the onset of electric breakdown is associated with any kind of crystal defect, the breakdown rate has an exponential dependence on $E^{2}$. Moreover, in case the magnetic contribution to the stress is proportional to the electric field at the surface, the model also predicts that as first approximation the breakdown rate in rf accelerating components has an exponential dependence on $E_{\mathrm{acc}}^{2}$, as given in Eq. (8). Contrary to the previous model [8] the current model also has a functional form for a temperature dependence.

Clearly in both the dc and rf cases the $R_{\mathrm{BD}}$ is in this model very strongly increasing with $E$, in agreement with the experimental observations. This can be understood physically by the fact that a tensile stress makes formation of defects with positive relaxation volume easier, and that the basic equation for defect formation is exponential [26,27].

\section{TESTS OF MODEL}

\section{A. Electric field dependence}

To test the model with respect to dependence of BDR on electric field, we fitted the parameters in $a$ and $\Delta V$ in Eq. (7) or (8) to experimental data on the breakdown rate using the Levenberg-Marquardt least-squares algorithm [35] implemented for fitting arbitrary functions following Ref. [36]. For fitting we used $T=300 \mathrm{~K}$.

As the model is directly relevant to breakdown under static electric fields, we tested it by fitting the data on breakdown probability measured at the dc setup at CERN $[16,18,21]$. The results are illustrated in Fig. 1, which also shows a fit of a power law $a E^{x}$. The fits can be considered

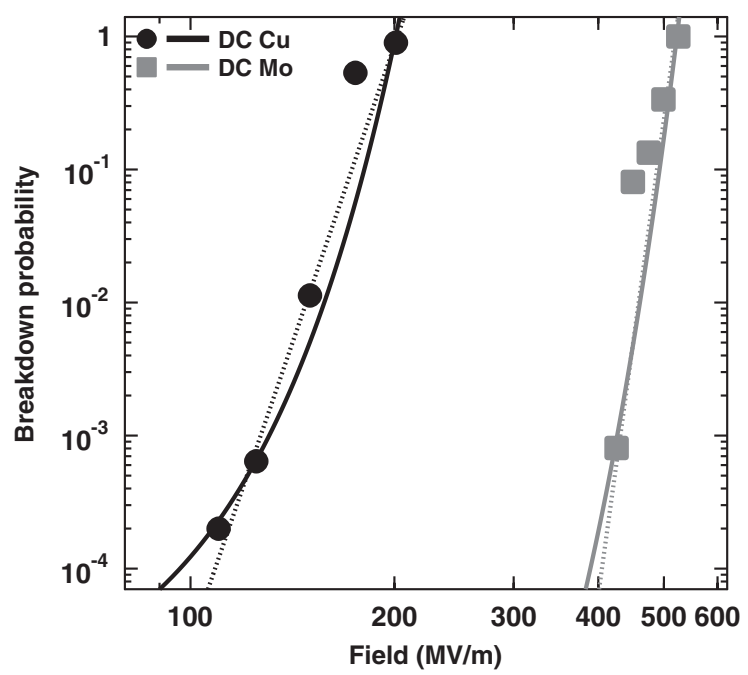

FIG. 1. Measured dependences of $R_{\mathrm{BD}}$ versus electric field at the dc breakdown measurement setup at CERN [21] and fits of our model (solid lines) as well as power laws (dashed lines) to the data.

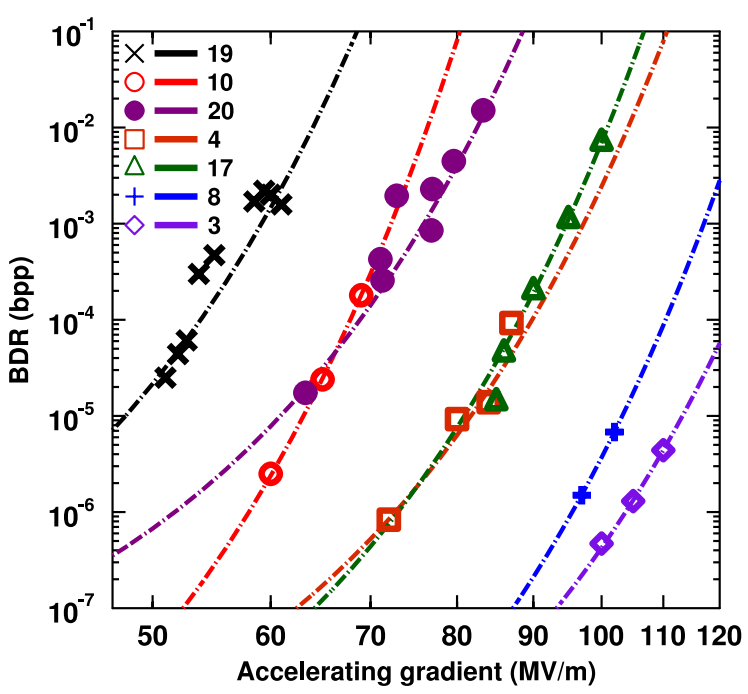

FIG. 2. Measured dependences of breakdown rate $R_{\mathrm{BD}}$ (in units of breakdown per pulse, bpp) versus accelerating gradient $E_{\text {acc }}$ for different accelerating structures [8] and fits of the model to the data. The numbers indicate the data sets as defined in Ref. [8].

to be of comparable quality to each other, showing that the model developed in this paper can also reproduce experimentally observed breakdown rates.

Since the power law model (a dependence on electric field only) has been used to examine breakdown rates in $\mathrm{rf}$ cavities, we proceeded to test whether the current model can also fit well $\mathrm{rf}$ breakdown rates. The results are illustrated in Figs. 2-4. Figures 2 and 3(a) show that the rf experimental data can be very well reproduced by the model. Figure 3(b) shows fits of a power law $a E^{x}$ to the data. Comparison of the fits in parts (a) and (b) show that the fits are of comparable quality. The data set "Circular Mo" is clearly better reproduced by the current model, however, considering the scatter in the other experimental data sets, this may simply be fortuitous.

The fit (which was carried out taking the errors into account [36]) in Fig. 4 shows that the current model provides a better fit to the highest gradient data point, which has the smallest error bars.

\section{B. Interpretation of $\Delta V$ values}

The fit results are given in Table I, in terms of the prefactor and $\Delta V \eta^{2}$. For dc experiments $\eta=1$. For $\mathrm{rf}$ components, analysis of the experimental conditions show [34] that for the electric field $\eta$ is in the range 1.86-2.13. Assuming a value of $\eta=2.0$, we can roughly estimate that the relaxation volumes $\Delta V$ are in the range $40-1100 \mathrm{~nm}^{3}$. Using an approximate value of $0.01 \mathrm{~nm}^{3}$ for the atomic volume $\Omega$ in metals (e.g. in $\mathrm{Cu}$ the true value is $0.0119 \mathrm{~nm}^{3}$ ), this translates to $\approx 4000-110000 \Omega$.

This size scale suggests a possible nature of the defects responsible for the breakdown. The tips believed to be 

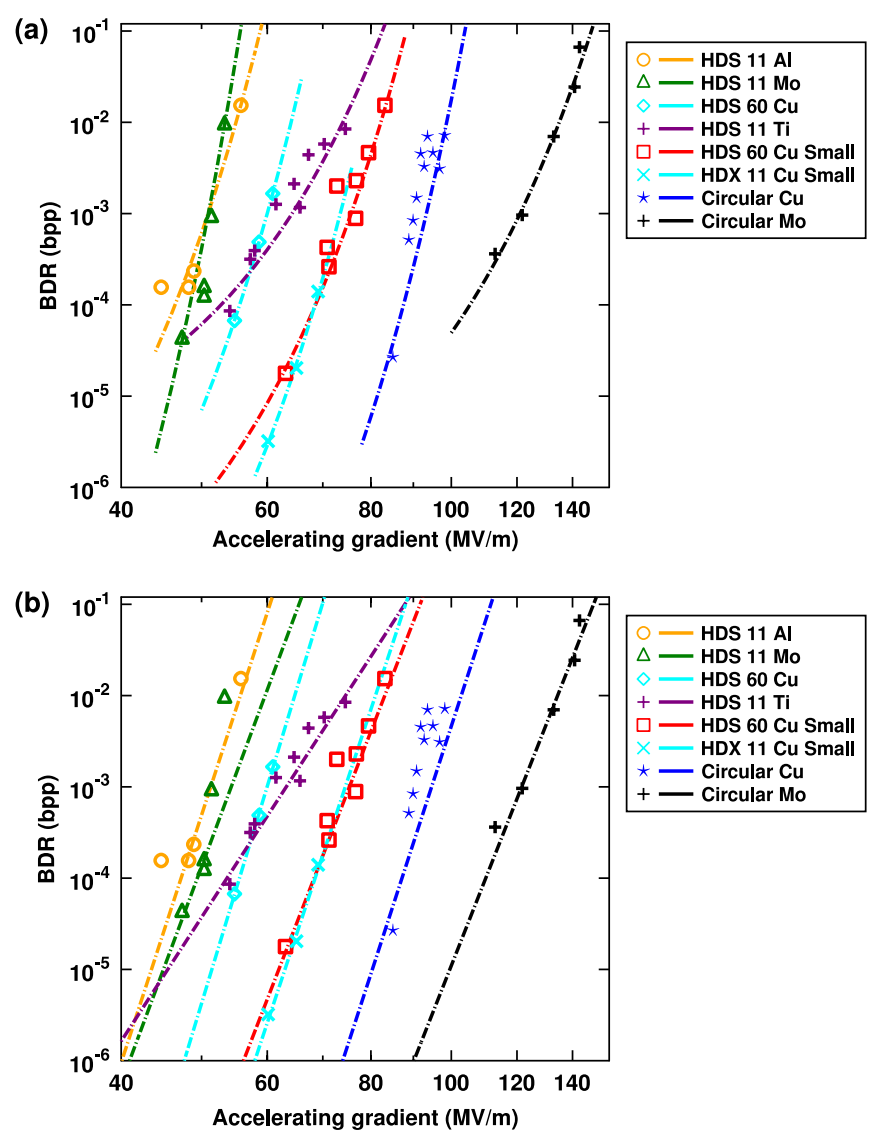

FIG. 3. (a) Measured dependences of $R_{\mathrm{BD}}$ (in units of breakdown per pulse, bpp) versus electric field for different accelerating structures and fits of the model to the data. For clarity, the results of the functional fit are not shown for all $E$ values for all data sets. (b) Fits of power law functions to the same data. The experimental data and their labels are from [42].

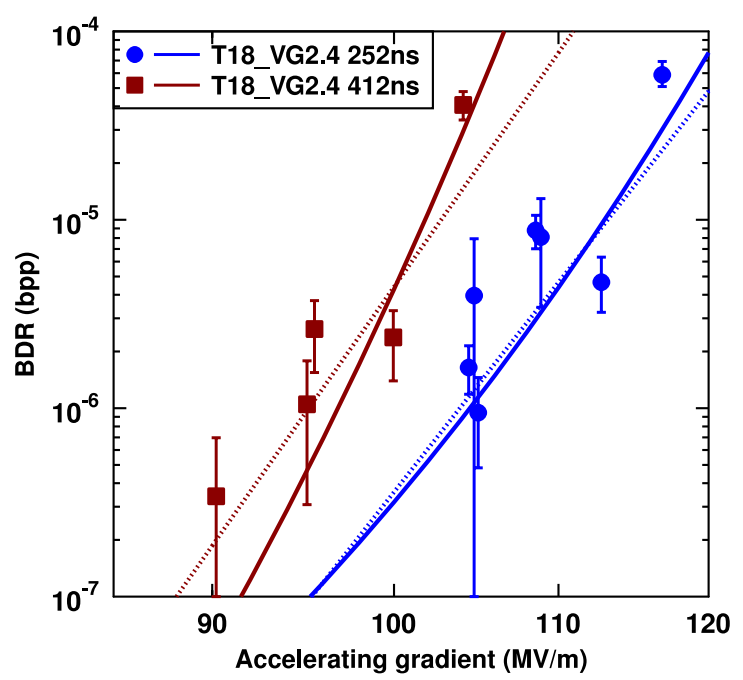

FIG. 4. Measured dependences of $R_{\mathrm{BD}}$ (in units of breakdown per pulse, bpp) versus electric field for the T18 accelerating structure $[33,43]$ and fits of our model (solid lines) as well as power laws (dashed lines) to the data.

TABLE I. Results from fit. The units of $a$ is breakdowns per pulse (bpp) and for $\Delta V m^{-3} . \eta$ is a unitless factor that gives the ratio between surface field and accelerating gradient (see text) in rf cavities. For the DC experiments (top two entries) $\eta=1$. The labels correspond to the ones in Figs. 1-4.

\begin{tabular}{lcc}
\hline \hline Label & $a(\mathrm{bpp})$ & $\Delta V \eta^{2}\left(\mathrm{~m}^{-3}\right)$ \\
\hline DC Cu & $0.632240 \times 10^{-05}$ & $0.138760 \times 10^{-24}$ \\
DC Mo & $0.101751 \times 10^{-08}$ & $0.354737 \times 10^{-25}$ \\
19 & $0.217011 \times 10^{-08}$ & $0.147812 \times 10^{-23}$ \\
10 & $0.246328 \times 10^{-10}$ & $0.131642 \times 10^{-23}$ \\
20 & $0.280939 \times 10^{-08}$ & $0.906207 \times 10^{-24}$ \\
4 & $0.780480 \times 10^{-13}$ & $0.113770 \times 10^{-23}$ \\
17 & $0.192000 \times 10^{-11}$ & $0.966961 \times 10^{-24}$ \\
8 & $0.219745 \times 10^{-12}$ & $0.739714 \times 10^{-24}$ \\
3 & $0.829316 \times 10^{-13}$ & $0.686151 \times 10^{-24}$ \\
HDS 11 Al & $0.109045 \times 10^{-08}$ & $0.247608 \times 10^{-23}$ \\
HDS 11 Mo & $0.501828 \times 10^{-13}$ & $0.427205 \times 10^{-23}$ \\
HDS 60 Cu & $0.885219 \times 10^{-10}$ & $0.211061 \times 10^{-23}$ \\
HDS 11 Ti & $0.849027 \times 10^{-06}$ & $0.800532 \times 10^{-24}$ \\
HDS 60 Cu small & $0.264894 \times 10^{-08}$ & $0.104744 \times 10^{-23}$ \\
HDX 11 Cu small & $0.236608 \times 10^{-10}$ & $0.152118 \times 10^{-23}$ \\
Circular Cu & $0.404675 \times 10^{-11}$ & $0.103809 \times 10^{-23}$ \\
Circular Mo & $0.763293 \times 10^{-07}$ & $0.302748 \times 10^{-24}$ \\
T18_VG2.4 252 ns & $0.119730 \times 10^{-11}$ & $0.584143 \times 10^{-24}$ \\
T18_VG2.4 412 ns & $0.389232 \times 10^{-15}$ & $0.108155 \times 10^{-23}$ \\
\hline \hline
\end{tabular}

grown on the surfaces are estimated to be in the size range $10-100 \mathrm{~nm}$ [8]. Such tips could, at least in principle, grow by emission of interstitial-type prismatic dislocation loops (platelets of extra atoms in the lattice) [37-39] or dislocation loops with the Burgers vector in the glide plane $[24,37,40]$ in the similar size range. Large interstitial-like dislocation loops in metals are known to have a relaxation volume of $\approx 1 \Omega$ per atom in the loop [31,40]. Assuming circular interstitial-like dislocation loops of one atom layer thickness, we can now roughly estimate the radius of one loop from $\pi r^{2} d=\Delta V$, where $d$ is the thickness of one atomic layer. Using a typical value of $d=0.2 \mathrm{~nm}$, one obtains a range of loop radii $\approx 8-40 \mathrm{~nm}$. This value compares very well with the experimental estimates of tip sizes $\sim 10-100 \mathrm{~nm}[8]$.

\section{Interpretation of power law exponents}

We next turn to the question of why the power law exponents obtained in previous fits are so high (typical dependences are about $E^{30}$ [8]). The fits obtained in Figs. 1-4 show that the slope of the two fitted functions

$$
\begin{gathered}
R_{\mathrm{BD}}=a e^{\varepsilon_{0} E^{2} \Delta V /\left(k_{B} T\right)} \\
R_{\mathrm{BD}}^{\prime}=a^{\prime} E^{\gamma}
\end{gathered}
$$

is similar in the $E$ range of interest. To compare the slopes as a function of $E$, we take the logarithm of both equations:

$$
\log R_{\mathrm{BD}}=\log a+\frac{\varepsilon_{0} E^{2} \Delta V}{k_{B} T}
$$




$$
\log R_{\mathrm{BD}}^{\prime}=\log a^{\prime}+\gamma \log E
$$

and make a Taylor series expansion around a field value $E_{0}$ in the range of interest, which gives

$$
\begin{gathered}
\log R_{\mathrm{BD}} \approx \log a+\frac{\varepsilon_{0} E_{0}^{2} \Delta V}{k_{B} T}+\frac{2 \varepsilon_{0} E_{o} \Delta V}{k_{B} T}\left(E-E_{0}\right) \\
\log R_{\mathrm{BD}}^{\prime} \approx \log a^{\prime}+\gamma \log E_{0}+\frac{\gamma}{E_{0}}\left(E-E_{0}\right) .
\end{gathered}
$$

Comparison of these two forms shows that the slope of the curves close to $E_{0}$ is given by the prefactors to the $\left(E-E_{0}\right)$ terms, which allows one to solve for the exponent $\gamma$ :

$$
\gamma=\frac{2 \varepsilon_{0} E_{o}^{2} \Delta V}{k_{B} T}
$$

Taking for instance the values of $\Delta V=0.3547 \times 10^{-25} \mathrm{~m}^{-3}$ obtained in the "DC Mo" fit (see Table I and Fig. 1), expanding around $E_{0}=500 \mathrm{MV} / \mathrm{m}$ and using $T=$ $300 \mathrm{~K}$, one obtains $\gamma=37.8$, which agrees very well with the power law fit shown in Fig. 1 which gave $\gamma_{\text {fit }}=$ 36.9. This comparison shows that the very high exponents obtained in the previous power law fits are consistent with the current model, and can be explained by taking into account the tensile stresses due to the electric field component.

\section{Dependence on surface heating}

Radio-frequency experiments [41] have shown that the breakdown rate also depends strongly on the maximum surface heating, see Fig. 5. The heating is presumed to be primarily due to the magnetic component of the field, and thus not directly dependent on the electric field component.

In our model, the breakdown rate depends exponentially on inverse temperature, as evident, e.g., from Eq. (6). Since the heating is exponential, it is reasonable to assume that

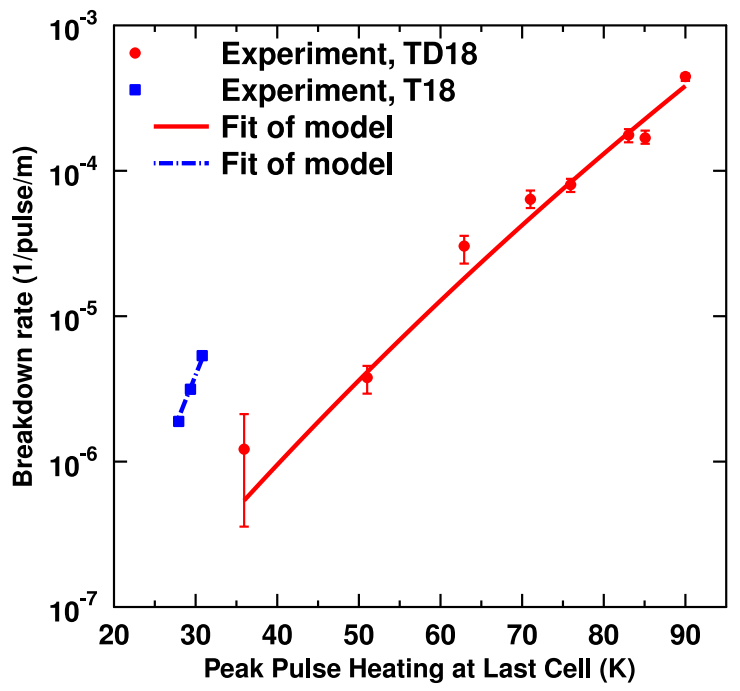

FIG. 5. Experimental data on dependence of breakdown rate on the peak temperature increase in accelerating components. The experimental data are from Ref. [41]. the breakdown rate is dominated by the regions of peak surface heating.

To analyze the experimental dependence on temperature, we rewrite $T=T_{0}+\Delta T$, where $\Delta T$ is the peak pulse heating. Regrouping the terms, one can write Eq. (6) as

$$
R_{\mathrm{BD}}=a^{\prime} c_{0} e^{\left(-E^{f}+\varepsilon_{0} E^{2} \Delta V\right) /\left[k_{B}\left(T_{0}+\Delta T\right)\right]} .
$$

In this form, we can fit the experimental $R_{\mathrm{BD}}$ vs $\Delta T$ data. The fits give excellent agreement with the experiments, see Fig. 5.

We used in the fitting a reference temperature $T_{0}=$ $300 \mathrm{~K}$ and $E=\eta E_{\text {acc }}=2 \times 110 \mathrm{MV} / \mathrm{m}$ based on data for the accelerating field provided in [41]. Note that since the dependence of $\Delta T$ is linear on both $E^{f}$ and $\Delta V$, it is not possible to distinguish between these two terms in Eq. (16). Hence, the exact choice of $E$ does not matter, as the fit only can give the sum $\left(E^{f}-\varepsilon_{0} E^{2} \Delta V\right)$. This quantity is the defect formation enthalpy $H^{f}$, and we obtain $\approx 1.4 \mathrm{eV}$ for it.

Finally, as a minor side point, we consider why the dependence of $\log R_{\mathrm{BD}}$ with $\Delta T$ appears linear in Fig. 5. Taking the logarithm on both sides of Eq. (16) and using for brevity the defect formation enthalpy $H^{f}=E^{f}-\varepsilon_{0} E^{2} \Delta V$, one obtains

$$
\begin{aligned}
\log R_{\mathrm{BD}} & =\log a^{\prime} c_{0}+\log e^{-H^{f} /\left[k_{B}\left(T_{0}+\Delta T\right)\right]} \\
& =\log \left(a^{\prime} c_{0}\right)+\frac{-H^{f}}{k_{B} T_{0}\left(1+\frac{\Delta T}{T_{0}}\right)} \\
& \approx \log \left(a^{\prime} c_{0}\right)+\frac{-H^{f}}{k_{B} T_{0}}-\frac{-H^{f}}{k_{B} T_{0}} \frac{\Delta T}{T_{0}},
\end{aligned}
$$

where the Taylor series approximation $1 /(1+\epsilon) \approx 1-\epsilon$ was used since currently $\Delta T / T \ll 1$. The defect formation enthalpy term $H^{f}=E^{f}-\varepsilon_{0} E^{2} \Delta V$ must be positive (otherwise defect formation would be spontaneous). Hence, the term $-H^{f}$ is negative, and considering the minus sign in front of the last term in Eq. (19), it predicts as a first approximation a linear increase of $\log R_{\mathrm{BD}}$ with $\Delta T$, fully consistent with the experimental observations.

\section{DISCUSSION AND CONCLUSION}

Our model provides a possible physical reason to the strong dependence of the breakdown rate on electric field. Moreover, the curvature of the functional form in the log$\log$ plots provides a way to test the model. Indeed, the very good fit of the model to the data sets " 4 " and "Circular Mo" in Figs. 2 and 3 suggests that such a curvature is indeed present. If experimental data becomes available in a wider range of $E$, this should provide a test of the model.

Grudiev et al. have pointed out that any exponential model has the feature of predicting a nonzero breakdown rate at zero field, which is obviously not physical [8]. The current model does predict a nonzero breakdown rate at 
$E=0$. However, its physical motivation provides a natural solution to this conundrum: the defect concentration is indeed nonzero even without any stress. But without any electric field, the surface charging and field electron emission is zero, rendering electric breakdown essentially impossible. On the other hand, this suggests that it might be relevant to extend the model with a term dependent on the electron field emission. We tested that a direct fit of the Fowler-Nordheim equation does not alone provide a good fit to the data because the function is fairly flat around the electric field region of interest; however, a product of the Fowler-Nordheim equation and the current model clearly would provide a good fit. However, since already the current model gives very good fits to the data, one cannot currently deduce further information from the fits, as more free parameters would lead to overfitting.

The surface heating analysis (Sec. III D) shows that the model is also consistent with temperature changes. However, one should note that the fits of electric field and heating are not completely independent. The magnetic field causing the heating is correlated with the electric field. The model could be extended to fit both variables at the same time, but as the fits are already essentially perfect with one variable fitting only, one could not (similarly to the case of the Fowler-Nordheim fits) deduce more information from such fits without additional experimental data.

Experience in building klystrons and high-gradient accelerating structures show that high temperature heat treatments, in excess of 1000 degrees, are extremely beneficial for improving the conditioning time (the number of breakdowns needed to achieve operating levels) and high-gradient performances of the devices. Our model also provides a tentative physical interpretation to this. Annealing reduces defect density in a material and increases grain size. Both effects reduce the possibilities to form dislocation-type defects, i.e., reduce the defect formation entropy in Eq. (4) and hence the expected defect concentration.

The current model is not able to explain all features of the complex breakdown phenomenon. However, a further refinement of the model can be done by incorporating the effect of the magnetic-field induced stresses. Similarly, the possible role of field enhancement factors $\beta$ of a tip is not currently included in the model. This factor will lead to a locally enhanced tensile stress, which could be accounted for by adding a $\beta$ factor with the field $E$. However, as $\beta$ is not known in the general case, we have not included it at this stage. Likewise, the dislocation mobility rates are not included in any way. However, since dislocations can move on picosecond time scales, we believe that the rate-limiting step in breakdowns is the initial nucleation, which is described by the formation enthalpy in the current model. If more accurate understanding of the breakdown triggering mechanism becomes available, the model could be extended to include also defect migration rates (which are described by another exponential term [30]).

Finally, we note that, although we assumed in the discussion above that the defects would be dislocations, the model does not per se require this: it is equally valid for any crystallographic defect. However, considering their dominating role in the plasticity of metals [27], dislocations are certainly the most likely candidate for the defect in the model.

In conclusion, we have presented a physically motivated model that can reproduce well a wide range of data on the dependence of vacuum microwave breakdown rate on electric field in high-gradient linear collider components.

\section{ACKNOWLEDGMENTS}

We thank T. Higo and A. Grudiev for providing their data set for our use, as well as A. Pohjonen, A. Grudiev, S. Calatroni, and V. Dolgashev and especially W. Wuensch for many useful discussions. This work was performed within the Finnish Centre of Excellence in Computational Molecular Science (CMS), financed by The Academy of Finland and the University of Helsinki, and was also supported by the Academy of Finland project MECBETH.

[1] The Compact Linear Collider Study, http://clic-study .web.cern.ch/clic-study/.

[2] C. Adolphsen, in Proceedings of the 2003 Particle Accelerator Conference, edited by J. Chew, P. Lucas, and S. Webber (IEEE, Washington, 2003), Vol. 1-5, pp. 668-672.

[3] C. Adolphsen, in Proceedings of the 2005 PAC Conference (IEEE, Washington, 2005) [http://accelconf.web.cern.ch/ Accelconf/p05/PAPERS/TOPE002.PS].

[4] R. Corsini, S. Döbert, R. Fandos, A. Grudiev, E. Jensen, T. Ramsvik, J. Rodriguez, J. Sladen, I. Syratchev, M. Taborelli, F. Tecker, P. Urschütz, I. Wilson, W. Wuensch, and O. Mete, in Proceedings of LINAC 2006 (2006), THP077.

[5] W. Wuensch, Technical Report No. CERN-AB-2006-085 CLIC Note 700, CERN (unpublished).

[6] D. R. Swenson, A. T. Wu, E. Degenkolb, and Z. Insepov, AIP Conf. Proc. 877, 370 (2006).

[7] A. Descoeudres, S. Calatroni, and M. Taborelli, in Proceedings of the 11th European Particle Accelerator Conference, Genoa, 2008 (EPS-AG, Genoa, Italy, 2008), p. 577.

[8] A. Grudiev, S. Calatroni, and W. Wuensch, Phys. Rev. ST Accel. Beams 12, 102001 (2009).

[9] F. Wang, C. Adolphsen, and C. Nantista, Phys. Rev. ST Accel. Beams 14, 010401 (2011).

[10] B. Jüttner, Beiträge aus der Plasmaphysik 19, 25 (1979).

[11] R. Behrisch, in Physics of Plasma-Wall Interactions in Controlled Fusion, NATO Advanced Study Institutes, Ser. B, Vol. 131, edited by D. E. Post and R. Behrisch (Plenum Press, New York, 1986), pp. 495-513. 
[12] H. Timkó, F. Djurabekova, L. Costelle, K. Nordlund, K. Matyash, R. Schneider, A. Toerklep, G. Arnau-Izquierdo, A. Descoeudres, S. Calatroni, M. Taborelli, and W. Wuensch, Phys. Rev. B 81, 184109 (2010).

[13] R. G. Forbes, Appl. Surf. Sci. 87/88, 1 (1995).

[14] H. H. Braun, S. Döbert, I. Syratchev, M. T. I. Wilson, and W. Wuensch, Technical Report No. CERN/PS 2002-062 (RF), CERN, Cern, Geneva (unpublished).

[15] A. Grudiev and W. Wuensch, in Proceedings of Linear Accelerator Conference LINAC2010 [http://epaper.kek.jp/ index.html], MOP068.

[16] T. Ramsvik, S. Calatroni, A. Reginelli, and M. Taborelli, Phys. Rev. ST Accel. Beams 10, 042001 (2007).

[17] A. Descoeudres, Y. Levinsen, S. Calatroni, M. Taborelli, and W. Wuensch, Phys. Rev. ST Accel. Beams 12, 092001 (2009).

[18] S. Calatroni, A. Descoeudres, A. Hansen, J. W. Kovermann, M. Taborelli, H. Timko, W. Wuensch, F. Djurabekova, K. Nordlund, A. Pohjonen, and A. Kuronen, in Proceedings of the 2010 LINAC conference [http://epaper.kek.jp/LINAC2010/papers/mop070.pdf].

[19] H. Timko, M. Aicheler, P. Alknes, S. Calatroni, A. Oltedal, A. Toerklep, M. Taborelli, W. Wuensch, F. Djurabekova, and K. Nordlund, Phys. Rev. ST Accel. Beams 14, 101003 (2011).

[20] A. Descoeudres, F. Djurabekova, and K. Nordlund, CLIC-Note 1 (2010).

[21] A. Descoeudres, T. Ramsvik, S. Calatroni, M. Taborelli, and W. Wuensch, Phys. Rev. ST Accel. Beams 12, 032001 (2009).

[22] J. Kovermann, Ph.D. thesis, RWTH Aachen, 2010.

[23] F. Djurabekova, S. Parviainen, A. Pohjonen, and K. Nordlund, Phys. Rev. E 83, 026704 (2011).

[24] A. S. Pohjonen, F. Djurabekova, A. Kuronen, K. Nordlund, and S. Fitzgerald, J. Appl. Phys. 110, 023509 (2011).

[25] J. D. Jackson, Classical Electrodynamics (John Wiley \& Sons, New York, 1975).
[26] F. Mandl, Statistical Physics (Wiley, Chichester, UK, 1988), 2nd ed.

[27] W. D. Callister, Jr., Materials Science and Engineering, An Introduction (Wiley, New York, 1993), 3rd ed.

[28] D.C. Wallace, Thermodynamics of Crystals (Dover, Mineola, NY, 1972).

[29] N. L. Peterson, J. Nucl. Mater. 69\&70, 3 (1978).

[30] P. Ehrhart, in Properties and Interactions of Atomic Defects in Metals and Alloys, Landolt-Börnstein, New Series, Group III, Vol. 25, edited by H. Ullmaier (Springer, Berlin, 1991), Chap. 2, p. 88.

[31] A. B. Sivak, V. A. Romanov, and V. M. Chernov, AIP Conf. Proc. 999, 118 (2008).

[32] K. Nordlund and R. S. Averback, Phys. Rev. Lett. 80, 4201 (1998).

[33] S. Matsumoto, T. Abe, Y. Higashi, T. Higo, and Y. Du, Nucl. Instrum. Methods Phys. Res., Sect. B 657, 160 (2011).

[34] W. Wuensch and A. Grudiev (private communication).

[35] W.H. Press, S. A. Teukolsky, W. T. Vetterling, and B. P. Flannery, Numerical Recipes in C; The Art of Scientific Computing (Cambridge University Press, New York, 1995), 2nd ed.

[36] P. R. Bevington, Data Reduction and Error Analysis for the Physical Sciences (McGraw-Hill, New York, 1992).

[37] D. Hull and D. J. Bacon, Introduction to Dislocations (Butterworth, Washington, DC, 2001), 4th ed.

[38] J.P. Hirth and J. Lothe, Theory of Dislocations (Krieger, Malabar, Florida, 1992), 2nd ed.

[39] B. L. Eyre, J. Phys. F 3, 422 (1973).

[40] S. Ryu, K. Kang, and W. Cai, J. Mater. Res. 26, 2335 (2011).

[41] F. Wang, C. Adolphsen, and C. Nantista, at the 2011 HighGradient Workshop [http://www-conf.slac.stanford.edu/ hg2011/talks/Feb9/Faya_USHG_2011.pptx].

[42] W. Wuensch [http://indico.cern.ch/conferenceDisplay.py? confId $=8831]$.

[43] Toshiyasu Higo (private communication). 